\title{
Acute Myeloid Leukemia with Translocation (8;16)(p11;p13): A Distinct Syndrome - Case Report and Literature Review
}

Naomi Dempsey ${ }^{1 *}$, Moh'd Khushmann², Peter Hosein ${ }^{1}$, Clifford Blieden ${ }^{3}$, Jennifer Chapman-Fredricks ${ }^{3}$ and Ronan Swords ${ }^{1}$

${ }^{1}$ University of Miami/Sylvester Cancer Center, Miami, USA

${ }^{2}$ University of South Alabama, USA

${ }^{3}$ University of Miami, Miami, USA

\begin{abstract}
Acute myeloid leukemia with the translocation $(8 ; 16)(p 11 ; p 13)$ is a rare type of acute leukemia with a number of unique features including erythrophagocytosis, extramedullary disease, and poor prognosis with high relapse rate. These cases of AML are often categorized as M4, M5a, or M5b AML under the FAB system of AML classification. However, the clinical and pathological features of $A M L$ with $t(8 ; 16)(p 11 ; p 13)$ do not fit into any of these FrenchAmerican-British (FAB) classification system subtypes, nor is it recognized as a recurrent genetic abnormality within the WHO classification system. Here, we report the case of a 50-year-old female with a history of low-grade carcinoid tumor on surveillance who developed de novo AML with histiocytic differentiation and $t(8 ; 16)(p 11 ; p 13)$. Immunohistochemistry and morphology of the patient's bone marrow biopsy was not consistent with any specific AML subtype, which resulted in diagnostic and therapeutic delays. AML with $t(8 ; 16)(\mathrm{p} 11 ; \mathrm{p} 13)$ has been described a number of times in the literature as a unique leukemic syndrome based on clinical, cytochemical, and DNA microarray features. As such, AML with $\mathrm{t}(8 ; 16)(\mathrm{p} 11 ; \mathrm{p} 13)$ should be added to the WHO classification system list of AML with recurrent genetic abnormalities.
\end{abstract}

Keywords: Acute myeloid leukemia with translocation $(8 ; 16)(\mathrm{p} 11 ; \mathrm{p} 13)$; Acute Myeloid Leukemia (AML); FAB classification system for AML; WHO classification system; AML with recurrent genetic abnormalities

\section{Introduction}

Acute myeloid leukemia (AML) encompasses a group of diseases caused by various mutations that lead to clonal expansion of dysfunctional hematopoietic progenitor cells called 'blasts.' The prognosis of AML depends on the cytogenetic abnormalities in the blasts, which define the disease. A number of specific mutations have been identified in the pathogenesis of AML. Some have even led to groundbreaking advances in treatment, as in the case of the translocation $(15 ; 17)$ mutation discovered in acute promyelocytic leukemia. Characterization of the genetic defects in these blasts allows clinicians to counsel their patients about the prognosis of their disease and the response they can expect from treatment [1].

AML with translocation $(8 ; 16)(\mathrm{p} 11 ; \mathrm{p} 13)$ is a rare subtype of AML that has been documented in the literature as a distinct syndrome. This translocation results in the fusion of MYST3 and CREB-binding protein gene. These gene products are involved in transcriptional regulation and cell cycle control, and they exhibit histone acetyltransferase activity. MYST3, in particular, serves as a co-activator for RUNX1 [2-4]. This AML syndrome is characterized by hemophagocytosis by the blasts, extramedullary disease manifestations, and poor prognosis. A study by Haferlach et al. reported a median overall survival of 4.7 months amongst adult patients diagnosed with AML with $t(8 ; 16)(\mathrm{p} 11 ; \mathrm{p} 13)$ [5]. The French-American-British morphology classification of AML cases with $\mathrm{t}(8 ; 16)(\mathrm{p} 11 ; \mathrm{p} 13)$ usually results in an M5 classification, but sometimes as M4 or M4/M5 variant [6-11]. The WHO classification system identifies a number of recurrent genetic abnormalities that are commonly seen in AML. AML with $\mathrm{t}(8 ; 16)(\mathrm{p} 11 ; \mathrm{p} 13)$ is not currently included in this list of recurrent genetic abnormalities [12]. A number of papers have proposed that AML with $\mathrm{t}(8 ; 16)(\mathrm{p} 11 ; \mathrm{p} 13)$ constitutes its own unique syndrome based on clinical features and immunophenotype [3,5,7,10,13,14]. Classification of AML with $t(8 ; 16)(\mathrm{p} 11 ; \mathrm{p} 13)$ in the current FAB system often becomes unclear when taking immunohistochemistry into account [2,5,7]. A new category of M5c AML has been proposed in the past to describe monocytic AML with malignant histiocytes demonstrating prominent hemophagocytosis. [13,15]. Perhaps AML with $t(8 ; 16)$ (p11;p13) is more adequately classified as M5c AML within the FAB classification or AML with recurrent genetic abnormalities within the WHO classification. Here we report a case of a 50-year-old woman with a history of surgically treated low-grade carcinoid on surveillance who presented with pancytopenia and epistaxis. She was ultimately diagnosed with acute monoblastic leukemia with translocation $(8 ; 16)$ (p11;p13).

\section{Case Report}

A 50-year-old Cuban female was diagnosed with low-grade carcinoid tumor of the jejunum in May of 2012, which was surgically resected. Margins were negative and lymph node biopsies done at that time were negative for carcinoid involvement. She presented to our center for follow up in August 2012, and CT scan of the abdomen showed a $1.8 \mathrm{~cm}$ calcific lymph node at the root of the mesentery. This finding was favored to represent local metastasis because it correlated with an area of increased uptake on Octreotide scan and the patient continued to have diarrhea with elevated HIAA- 5 levels. The case was presented in tumor board, and it was determined that surgical resection of the metastatic lymph node would not be appropriate because resection would have required major vascular surgical reconstruction. Since the tumor was low-grade, surveillance with serum markers and imaging was performed and demonstrated no growth. No cytotoxic chemotherapy or radiation was given.

*Corresponding author: Dr. Naomi Dempsey, University of Miami/Sylvester Cancer Center, 1611 NW 12th Ave. Miami, FL 33136, USA, Tel: 305-965-1864; E-mail: naomidempseymd@gmail.com

Received: August 09, 2017; Accepted: August 26, 2017; Published: August 30, 2017

Citation: Dempsey N, Khushmann M, Hosein P, Blieden C, Chapman-Fredricks J, et al. (2017) Acute Myeloid Leukemia with Translocation (8;16)(p11;p13): A Distinct Syndrome- Case Report and Literature Review. Oncol Cancer Case Rep 3: 134.

Copyright: () 2017 Dempsey N, et al. This is an open-access article distributed under the terms of the Creative Commons Attribution License, which permits unrestricted use, distribution, and reproduction in any medium, provided the original author and source are credited. 
Fourteen months after the diagnosis of carcinoid tumor, the patient developed epistaxis, fatigue and exertional shortness of breath. Pancytopenia was discovered on routine blood work and she was admitted. She had an absolute neutrophil count of less than 200, developed neutropenic fever, and required antibiotic treatment and intensive care. Her platelets nadired at $16 \mathrm{~K}$ and her hemoglobin nadired at $6.8 \mathrm{~g} / \mathrm{dl}$, necessitating transfusion support.

Bone marrow biopsy and aspirate revealed an acute monoblastic leukemia with histiocytic differentiation, hemophagocytosis, and presence of translocation $(8 ; 16)(\mathrm{p} 11 ; \mathrm{p} 13)$ in a background of myelodysplasia without evidence of metastatic carcinoid tumor. The patient underwent standard induction therapy with $7+3$ and achieved remission. Due to suboptimal performance status, consolidation with high dose ARA-C was undertaken as opposed to allergenic stem cell transplant. The patient's disease ultimately returned, and she opted to receive palliative Vidaza, but she did not respond and it was discontinued. She went on to receive hospice care, and passed at home with her family.

\section{Results}

Bone marrow aspirates stained with traditional Wright-Giemsa methodology were notable for abundant large monoblasts (Figure 1) with abnormal chromatin, increased nuclear to cytoplasmic features, and marked erythrophagocytosis (Figure 2). Histologic sections of the bone marrow core biopsy demonstrated involvement by blasts positive for CD45, CD4, CD68, and muramidase, confirming the monocytic lineage of the tumor. CD34, CD117, and MPO were negative. Peripheral blood smear was negative for blasts. Metastatic carcinoid was not identified. Conventional cytogenetics identified a complex karyotype $(45, \mathrm{XX}, \mathrm{t}(6 ; 12)(\mathrm{q} 21 ; \mathrm{q} 12), \mathrm{t}(8 ; 16)(\mathrm{p} 11 ; \mathrm{p} 13), \operatorname{der}(8) \operatorname{dic}(8 ; 18)(\mathrm{q} 22 ; \mathrm{p} 11.2)$ $\operatorname{trp}(80(\mathrm{q} 11.2 \mathrm{q} 22)[\mathrm{cp} 14] / 46, \mathrm{XX}(3))$ (Figure 2).

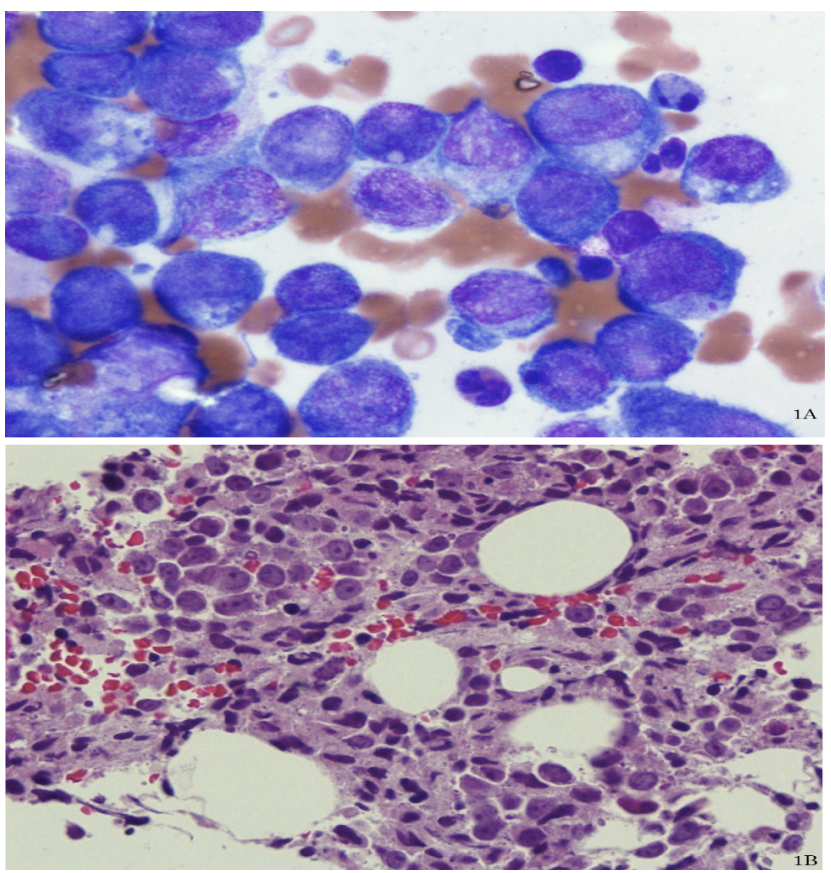

Figure 1: Bone marrow examination: $(1 \mathrm{~A})$ High power bone marrow aspirate showing blasts which were intermediate to large in size with monocytic features. (1B) Low power bone marrow core biopsy showing prominent monoblasts involving the bone marrow. Metastatic carcinoma was not present.
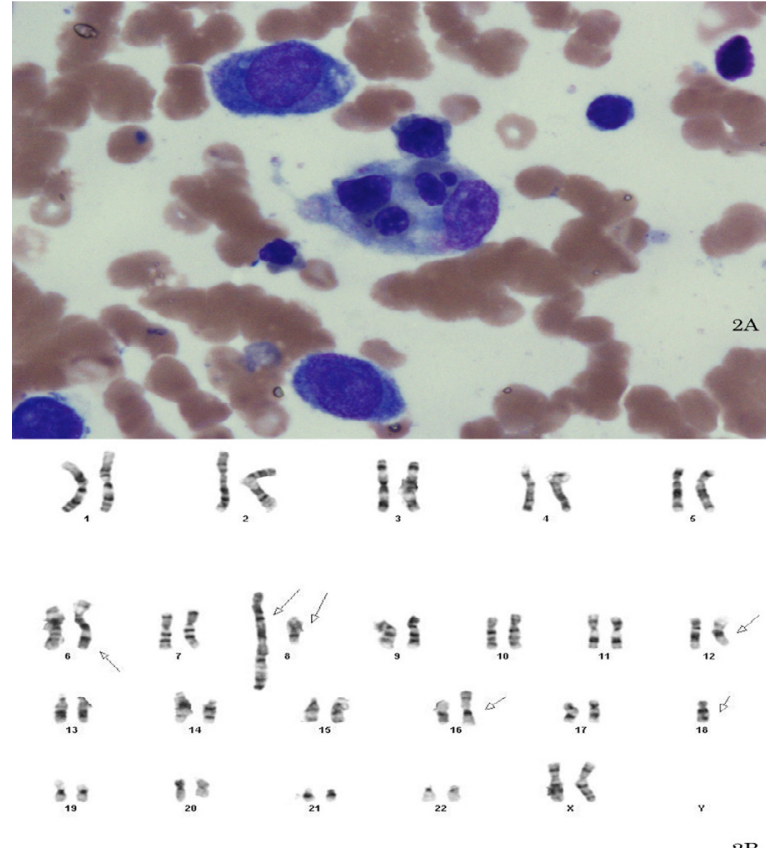

$2 \mathrm{~B}$

Figure 2: Diagnostic features: $(2 \mathrm{~A})$ High power bone marrow aspirate was notable for abundant monoblasts with marked erythrophagocytosis. (2B) Conventional cytogenetics identified a complex karyotype with $\mathrm{t}(8 ; 16)$ (p11;p13)

\section{Discussion}

Cases of AML with $\mathrm{t}(8 ; 16)(\mathrm{p} 11 ; \mathrm{p} 13)$ which have been reported in the literature have a number of common themes. Erythrophagocytosis, in which the leukemic blasts ingest red blood cells, is frequently seen in AML with $\mathrm{t}(8 ; 16)(\mathrm{p} 11 ; \mathrm{p} 13)$. In the case series by Diab et al. of 13 patients with AML with $\mathrm{t}(8 ; 16)(\mathrm{p} 11 ; \mathrm{p} 13), 7$ had blasts which exhibited erythrophagocytosis [2]. Amongst pediatric AML with $t(8 ; 16)$ (p11;p13), as many as 70\% of cases reported show erythrophagocytosis [3]. Erythrophagocytosis is a relatively rare finding in other types of AML, so this characteristic should raise concern for $t(8 ; 16)(\mathrm{p} 11 ; \mathrm{p} 13)$ as the culprit mutation. In the patient's case reported here, prominent erythrophagocytosis was identified. However, other characteristics of this case differed from other cases reported in the literature. AML with $\mathrm{t}(8 ; 16)(\mathrm{p} 11 ; \mathrm{p} 13)$ may commonly be associated with prior therapy for other malignancies, with breast being most frequent. Although this patient had a history of carcinoid tumor, she was managed surgically and never received any chemoradiation. Extramedullary manifestations are also commonly identified in AML with $\mathrm{t}(8 ; 16)(\mathrm{p} 11 ; \mathrm{p} 13)$. Leukemia cutis has been found in as many as $44 \%$ in Diab's case series. Other extramedullary manifestations included leptomeningeal, liver, and genitourinary involvement [2]. The patient in the case described here had none of these extramedullary findings, distinguishing her from other cases reported in the literature.

AML with $\mathrm{t}(8 ; 16)(\mathrm{p} 11 ; \mathrm{p} 13)$ produces a distinctive syndrome with immunophenotypic features that do not fit any of the currently categorized AML subtypes within the FAB classification system or within any of the recurrent genetic abnormalities in the WHO system. Cases of AML with $t(8 ; 16)(\mathrm{p} 11 ; \mathrm{p} 13)$ have historically been classified as M4, M5, or M4/M5 variant [6]. A study by Haferlach et al. performed a DNA microarray analysis of four cases of AML with $t(8 ; 16)(p 11 ; p 13)$, and compared them to microarrays of M1 $(n=46), M 4(n=41), M 5 a$ $(n=9)$, and M5b $(n=16)$ AML. The principle component analysis of 
gene expression microarrays revealed that AML with $t(8 ; 16)(\mathrm{p} 11 ; \mathrm{p} 13)$ clustered into its own group near but separate from M5a and M5b AML [5]. These results reinforce that AML with $t(8 ; 16)(\mathrm{p} 11 ; \mathrm{p} 13)$ may be an M5 AML, but that it does not neatly fit within either the FAB M5a or M5b categories. DNA microarrays can also be used to distinguish AML with $\mathrm{t}(8 ; 16)(\mathrm{p} 11 ; \mathrm{p} 13)$ from all other subtypes of AML. A study by Diaz-Beya et al. classified 120 different cases of AML of all subtypes according to their miRNA expression patterns. Seven of these $120 \mathrm{AML}$ cases were AML with $\mathrm{t}(8 ; 16)(\mathrm{p} 11 ; \mathrm{p} 13)$, and the miRNA signature of AML with $t(8 ; 16)(\mathrm{p} 11 ; \mathrm{p} 13)$ clustered tightly and independently from all other types of AML, including the other monocytic leukemias [14]. These findings suggest that AML with $t(8 ; 16)(\mathrm{p} 11 ; \mathrm{p} 13)$ represents a distinct entity which should be recognized as a recurrent genetic abnormality.

Khalidi et al. published a study that classified each type of AML within the FAB system based on its immunophenotypic features. M5 AML generally lacks CD34 expression, distinguishing M5 from all other subcategories of AML. M5a AML cases were usually negative for MPO and CD4, but positive for CD117. M5a (monoblastic) leukemia also tended to have a predominance of blasts in the bone marrow. M5b (monocytic) leukemia has far fewer blasts in the marrow and is variable in its expression for MPO, positive for CD4, and negative for CD117.CD4 and CD117 were the only markers found in this study to be statistically significant for distinguishing between M5a and M5b [16]. Our patient, like other M5 AML cases studied, was negative for CD34. The malignant blasts in the marrow were negative for MPO, which is consistent with either M5a or M5b. Her blast cells did not express CD117 but did express CD4, which is consistent with an M5b immunophenotype. However, the bone marrow sample had a predominance of blasts at $60 \%$ of all cells in the marrow prior to treatment. This high prevalence of blasts in the bone marrow resulted in a diagnosis of acute monoblastic leukemia. The patient's pathological findings do not neatly fit within either the M5a or M5b FAB AML classifications; the result was a delay in diagnosis and treatment. Although there is no targeted treatment for AML with $\mathrm{t}(8 ; 16)(\mathrm{p} 11 ; \mathrm{p} 13)$ yet, it is important to develop and utilize an accurate and inclusive classification system that allows for expedient diagnosis of AML subtypes so that treatment can be promptly initiated. The pathological features did not unambiguously fit any FAB subtype and definitive diagnosis based on immunophenotype and morphology was not possible. Diagnosis and treatment were delayed until cytogenetics confirmed translocation $(8 ; 16)(\mathrm{p} 11 ; \mathrm{p} 13)$. This genetic abnormality has historically been difficult to classify within the FAB system. Two possible alterations could alleviate this difficulty: the addition of the previously proposed M5c subtype to include AML with $\mathrm{t}(8 ; 16)(\mathrm{p} 11 ; \mathrm{p} 13)$, or the addition of AML with $\mathrm{t}(8 ; 16)(\mathrm{p} 11 ; \mathrm{p} 13)$ to the WHO category of AML with recurrent genetic abnormalities.

Many authors caution that diagnoses should not solely rest on immunophenotypic features because they can often overlap or contradict the remainder of the clinical picture. AML with $\mathrm{t}(8 ; 16)(\mathrm{p} 11 ; \mathrm{p} 13)$ has a very unique clinical/pathological picture and a poor prognosis, which has resulted in several proposals for a specific subclassification within the FAB system for this entity. As described above, the striking erythrophagocytosis displayed by blasts in AML with $\mathrm{t}(8 ; 16)(\mathrm{p} 11 ; \mathrm{p} 13)$ has been discussed as a distinctive feature numerous times in the literature $[2-7,9,10,14]$. The breakpoint observed in AML with $t(8 ; 16)$ (p11;p13) involves genes that are central to erythropoiesis. MYST3 acts as a co-activator of a number of hematopoietic transcription factors like RUNX1 and NF- $\kappa B$, and CREBBP plays an essential role in regulation of hematopoietic stem cell proliferation [14]. Mutations in RUNX1 have been recognized as a provisional entity in the WHO Classification causing an AML with particularly poor prognosis [12]. AML with $\mathrm{t}(8 ; 16)(\mathrm{p} 11 ; \mathrm{p} 13)$ disrupts the functioning of MYST3, which in turn will interrupt the proper functioning of RUNX1 by removing its coactivator. Dysfunction of these genes results in the erythrophagocytosis observed in $75 \%$ of $\mathrm{t}(8 ; 16)(\mathrm{p} 11 ; \mathrm{p} 13)$ cases, compared to hemophagocytosis present in $1 \%$ of all other AML subtypes $[4,9,14]$. Although non-specific, the presence of hemophagocytosis should raise suspicion for AML with $\mathrm{t}(8 ; 16)(\mathrm{p} 11 ; \mathrm{p} 13)$, even before genetic analysis or immunohistochemistry results become available. This is clinically important, because these patients have poor prognosis and a high rate of death from DIC, which may also occur long before genetic analysis results have returned. Early recognition of this clinical entity and a morphologic finding of erythrophagocytosis should raise our suspicion for AML with $\mathrm{t}(8 ; 16)(\mathrm{p} 11 ; \mathrm{p} 13)$ and prompt a search for signs of DIC as early intervention is key.

\section{Conclusion}

In conclusion, $A M L$ with $t(8 ; 16)(\mathrm{p} 11 ; \mathrm{p} 13)$ is a rare yet distinct clinical and pathological entity that cannot be adequately classified according to the current FAB system or WHO classification system. The M5c subtype has been proposed for leukemia with histiocytic differentiation, which includes AML with $t(8 ; 16)(p 11 ; p 13)$. The addition of AML with $\mathrm{t}(8 ; 16)(\mathrm{p} 11 ; \mathrm{p} 13)$ to the category of AML with recurrent genetic abnormalities has also been proposed, which could be adapted into both the FAB and WHO systems. Either of these categorical distinctions could eliminate a great deal of diagnostic difficulty, yielding a faster diagnosis and more immediate commencement of the indicated treatment regimes. Further research into the Myst3-CREBBP genes disrupted by $t(8 ; 16)(\mathrm{p} 11 ; \mathrm{p} 13)$ is warranted. The gene-targeted approach to the treatment of APL transformed a devastating disease into a curable leukemia. A similar treatment regime is theoretically possible for AML with $t(8 ; 16)(\mathrm{p} 11 ; \mathrm{p} 13)$ and, because of the very poor prognosis, should be vigorously pursued.

\section{References}

1. Estey E, Döhner H (2006) Acute myeloid leukemia. Lancet 368: 1894-1907.

2. Diab A, Zickl L, Abdel-Wahab O, Jhanwar S, Gulam MA, et al. (2013) Acute myeloid leukemia with translocation $\mathrm{t}(8 ; 16)$ presents with features which mimic acute promyelocytic leukemia and is associated with poor prognosis. Leuk Res 37: $32-36$

3. Coenen EA, Zwaan CM, Reinhardt D, Harrison CJ, Haas OA, et al. (2013) Pediatric acute myeloid leukemia with $\mathrm{t}(8 ; 16)(\mathrm{p} 11 ; \mathrm{p} 13)$ : A distinct clinical and biological entity, a collaborative study by the International-Berlin-FrankfurtMunster AML-study group. Blood 122: 2704-2713.

4. Giles RH, Dauwerse JG, Higgins C, Petrij F, Wessels JW, et al. (1997) Detection of CBP rearrangements in acute myelogenous leukemia with $\mathrm{t}(8 ; 16)$ Leukemia 11: 2087-2096.

5. Haferlach T, Kohlmann A, Klein HU, Ruckert C, Dugas M, et al. (2009) AML with translocation $\mathrm{t}(8 ; 16)(\mathrm{p} 11 ; \mathrm{p} 13)$ demonstrates unique cytomorphological, cytogenetic, molecular, and prognostic features. Leukemia 23: 934-943.

6. Stark B, Resnitzky P, Jeison M, Luria D, Blau O, et al. (1995) A distinct subtype of M4/M5 acute myeloblastic leukemia (AML) associated with $t(8: 16)(p 11: p 13)$, in a patient with the variant $t(8: 19)(p 11: q 13)$ - Case report and review of the literature. Leuk Res 19: 367-379.

7. Laï JL, Zandecki M, Jouet JP, Savary JB, Lambiliotte A, et al. (1987) Three cases of translocation $(8 ; 16)(\mathrm{pll} ; \mathrm{pl} 3)$ observed in acute myelomonocytic leukemia: A new specific subgroup? Cancer Genetics and Cytogenetics 27 : 101-109.

8. Heim S, Avanzi GC, Billström R, Kristoffersson U, Mandahl N, et al. (1987) A new specific chromosomal rearrangement, $\mathrm{t}(8 ; 16)(\mathrm{pll} ; \mathrm{pl} 3)$, in acute monocytic leukaemia. Brit J Haematol 66: 323-326.

9. Becher R, Haas OA, Graeven U, Bettelheim P, Ambros P, et al. (1988) Translocation $\mathrm{t}(8 ; 16)$ in acute monocytic leukemia. Cancer Genet Cytogenet 34: $265-271$ 
Citation: Dempsey N, Khushmann M, Hosein P, Blieden C, Chapman-Fredricks J, et al. (2017) Acute Myeloid Leukemia with Translocation (8;16)(p11;p13): A Distinct Syndrome- Case Report and Literature Review. Oncol Cancer Case Rep 3: 134.

10. Powell BL, McNay JW, Brown S, Cooper MR, Pettenati MJ (1988) Translocation $(8 ; 16)(\mathrm{p} 11 ; \mathrm{p} 13)$ in patients with acute monocytic leukemias. An evolving syndrome? Cancer Genet Cytogenet 36: 109-115.

11. Mitelman F, Heim S (1992) Quantitative acute leukemia cytogenetics. Genes, Chromosomes, and Cancer 5: 57-66.

12. Arber DA, Orazi A, Hasserjian R, Thiele J, Borowitz MJ, et al. (2016) The 2016 revision to the World Health Organization (WHO) classification of myeloid neoplasms and acute leukemia. Blood 127: 2391-2405.

13. Laurencet FM, Chapuis B, Roux-Lombard, Dayer JM, Beris $P$ (1993) Malignant histiocytosis in the leukaemic stage: $A$ new entity (M5c-AML) in the $F A B$ Classification? Leukemia 8: 502-506.
14. Díaz-Beyá M, Navarro A, Ferrer G, Diaz T, Gel B, et al. (2013) Acute myeloid leukemia with translocation $(8 ; 16)(\mathrm{p} 11 ; \mathrm{p} 13)$ and Myst3-CREBBP rearrangement harbors a distinctive microRNA signature targeting RET protooncogene. Leukemia 27: 595-603.

15. Esteve J, Rozman M, Campo E, Munoz F, Urbano-Ispizua A, et al. (1995) Leukemia after true histiocytic lymphoma: Another type of acute monocytic leukemia with histiocytic differentiation (AML-M5c)? Leukemia 9: 1389-1391.

16. Khalidi HS, Medeiros LJ, Chang KL, Brynes RK, Slovak ML, et al. (1998) The immunophenotype of adult acute myeloid leukemia: High frequency of lymphoid antigen expression and comparison of immunophenotype, FrenchAmerican-British classification, and karyotypic abnormalities. Am J Clin Patho 109: $211-220$. 